\title{
Treatment-resistant Lennox-Gastaut syndrome: therapeutic trends, challenges and future directions
}

\author{
This article was published in the following Dove Press journal: \\ Neuropsychiatric Disease and Treatment \\ 20 April 2017 \\ Number of times this article has been viewed
}

\author{
Adam P Ostendorf' \\ Yu-Tze $\mathrm{Ng}^{2}$ \\ 'Department of Pediatrics, Neurology \\ Section, Nationwide Children's \\ Hospital, The Ohio State University, \\ Columbus, $\mathrm{OH},{ }^{2}$ Department of \\ Pediatrics, Baylor College of Medicine, \\ The Children's Hospital of San \\ Antonio, San Antonio, TX, USA
}

\begin{abstract}
Lennox-Gastaut syndrome is a severe, childhood-onset electroclinical syndrome comprised of multiple seizure types, intellectual and behavioral disturbances and characteristic findings on electroencephalogram of slow spike and wave complexes and paroxysmal fast frequency activity. Profound morbidity often accompanies a common and severe seizure type, the drop attack. Seizures often remain refractory, or initial treatment efficacy fades. Few individuals are seizure free despite the development of multiple generations of antiseizure medications over decades and high-level evidence on several choices. Approved medications such as lamotrigine, topiramate, rufinamide, felbamate and clobazam have demonstrated efficacy in reducing seizure burden. Cannabidiol has emerged as a promising investigational therapy with vast social interest yet lacks a standard, approved formulation. Palliative surgical procedures, such as vagal nerve stimulation and corpus callosotomy may provide reduction in total seizures and drop attacks. Emerging evidence suggests that complete callosotomy provides greater improvement in seizures without additional side effects. Etiologies such as dysplasia or hypothalamic hamartoma may be amenable for focal resection and thus offer potential to reverse this devastating epileptic encephalopathy.
\end{abstract}

Keywords: Lennox-Gastaut syndrome, epilepsy, epilepsy surgery, cannabidiol, epileptic encephalopathy

\section{Introduction}

Five decades ago, Gastaut et al $^{1}$ summarized a clinical syndrome manifested as a "childhood epileptic encephalopathy with diffuse slow spikes-and-waves". Later termed as LennoxGastaut syndrome (LGS), this devastating epileptic encephalopathy remains one of the most treatment-resistant epilepsies across the age spectrum. LGS is currently classified by the International League Against Epilepsy (ILAE) as a childhood-onset electroclinical syndrome and epileptic encephalopathy comprised by the triad of, 1) polymorphic intractable seizures that are mainly tonic, atonic and atypical absence seizures; 2) cognitive and behavioral abnormalities; and 3) electroencephalogram (EEG) with paroxysms of fast activity and slow $(<2.5 \mathrm{~Hz})$ generalized spike wave discharges (Figure 1). ${ }^{2,3}$

The syndrome occurs in 2.8 per 100,000 live births ${ }^{4}$ and comprises $\sim 4 \%-10 \%$ of childhood epilepsy..$^{5-7}$ Onset is typically between 1 and 7 years and is five times more common in boys. ${ }^{5}$ Prognosis is poor, as less than $10 \%$ achieve seizure freedom as adults. ${ }^{8}$ Most individuals with LGS develop comorbid autism, intellectual disability and other behavioral concerns. ${ }^{9}$ Attention to these significant comorbidities is crucial in improving the quality of life in individuals with LGS; yet, no treatment specific to patients with LGS exists for these concerns. Patients' families have reported that their most significant concerns are fear of dying and the unpredictability of seizures, side effects and social isolation. ${ }^{10,11}$ These data may suggest prioritizing seizure control and facilitating interactions with appropriate support networks. 

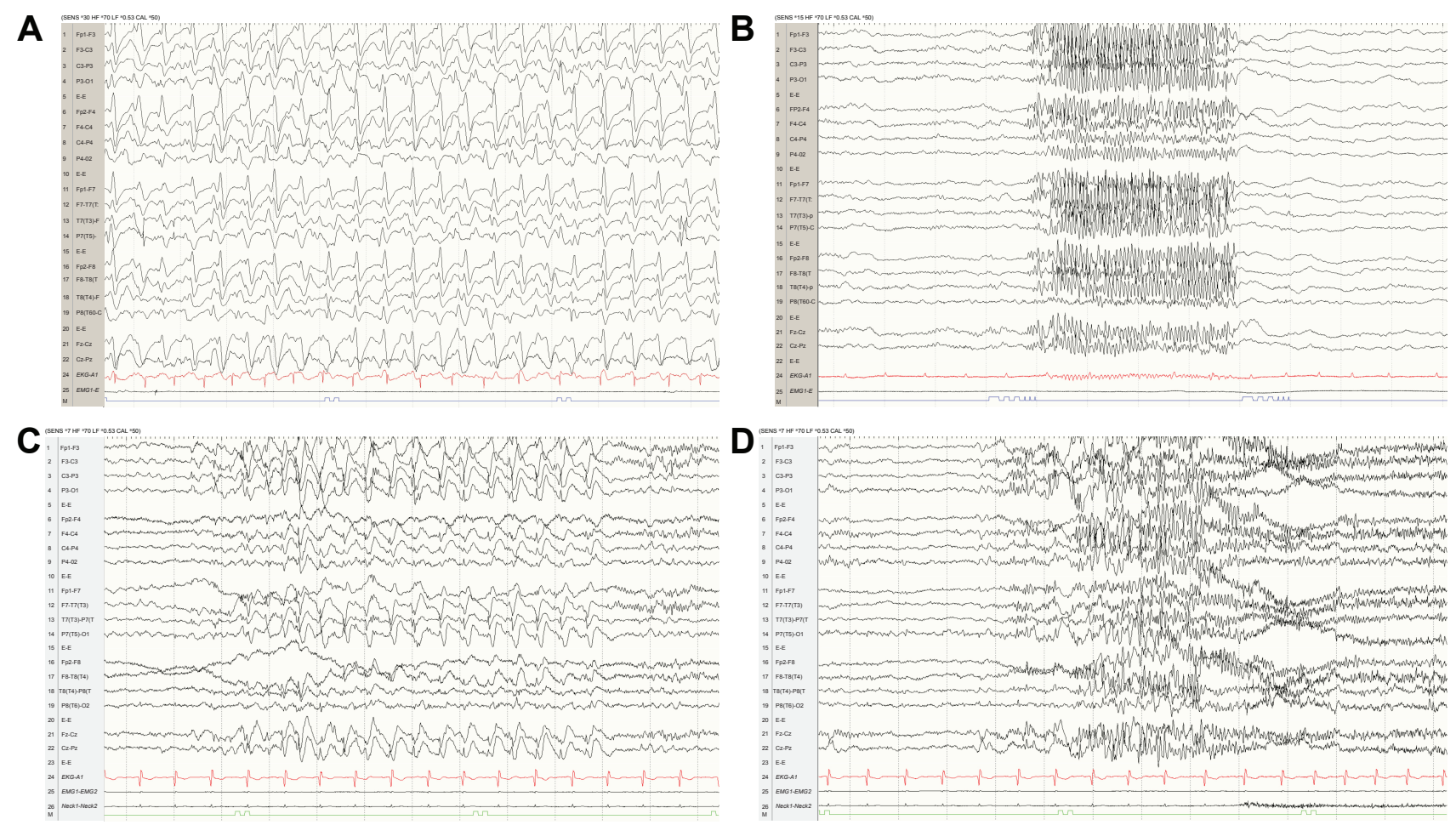

Figure I Electroencephalographic findings in Lennox-Gastaut syndrome.

Notes: Individuals with Lennox-Gastaut syndrome typically exhibit (A) generalized or diffuse slow spike and wave complexes and (B) generalized paroxysmal fast activity. (C) Lateralized predominance of epileptiform activity may be indicative of a focal lesion. (D) Generalized tonic seizures may arise from the background abnormalities.

Features portending a poor prognosis include preexisting West syndrome, early age of onset, symptomatic causes, abnormal neuroimaging, more frequent seizures or status epilepticus or focal/multifocal EEG abnormalities. ${ }^{5}$ Importantly, individuals with LGS have many risk factors for sudden unexplained death in epilepsy (SUDEP), such as frequent generalized tonic clonic seizures, early age of epilepsy, long duration of epilepsy and intellectual disability. ${ }^{12}$ Early death occurs in up to $15 \%$ of individuals with LGS. ${ }^{5}$

An extremely heterogeneous set of causes lead to a common syndrome in individuals with LGS. Structural etiologies, including hypoxic-ischemic encephalopathy, meningoencephalitis, neurocutaneous disorders and brain malformations, including hypothalamic hamartomas and metabolic syndromes, account for $\sim 70 \%-80 \%$ of all cases. ${ }^{13-15}$ Presumed genetic or unknown causes represent the other $20 \%-30 \% .^{14,16}$ De novo mutations in $A L G 13$ and GABRB3 have been described as causative in individuals with LGS. ${ }^{17}$ Other genes implicated include $C H D 2, D N M 1$, CACNA1A, CHD2, FLNA, GABRA1, GRIN1, GRIN2A, GRIN2B, HDAC3, HNRNPU, IQSEC2, mTOR, NEDD4L and $S C N 8 A, S T X B P 1$ and $S Y N G A P 1 .{ }^{18}$ Infantile spasms precede the development of LGS in up to $60 \%$ of children, more often if West syndrome was symptomatic., ${ }^{79-21}$

\section{Medical therapies - general principles and challenges}

Despite decades of medical advances, controlling seizures in patients with LGS remains a challenge for the clinician. More than $90 \%$ of children with LGS have drug-resistant epilepsy. ${ }^{22}$ Balancing seizure control and side effects using rational polypharmacy is challenging. While some combinations of antiseizure medications demonstrate therapeutic synergies, additive side effects can occasionally worsen seizures and often cause behavioral problems and increased sedation. ${ }^{5}$ Direct comparative drug trials in patients with LGS have not been performed. Six antiseizure medications hold US Food and Drug Administration (FDA) indications for the treatment of LGS, which include lamotrigine, topiramate, felbamate, rufinamide, clobazam and clonazepam. Notwithstanding multiple treatment options, long-term seizure control remains elusive. ${ }^{11}$

Comparing treatment efficacy in patients with LGS is challenging due to several factors. ${ }^{5}$ The reduction in seizures in individuals with LGS tends to diminish over time. ${ }^{5}$ Long-term follow-up for more than 12 months in prospective studies is often expensive and logistically challenging. The heterogeneity of seizure types in studies can be confounding, particularly the often-reported drop seizures. Most drop 


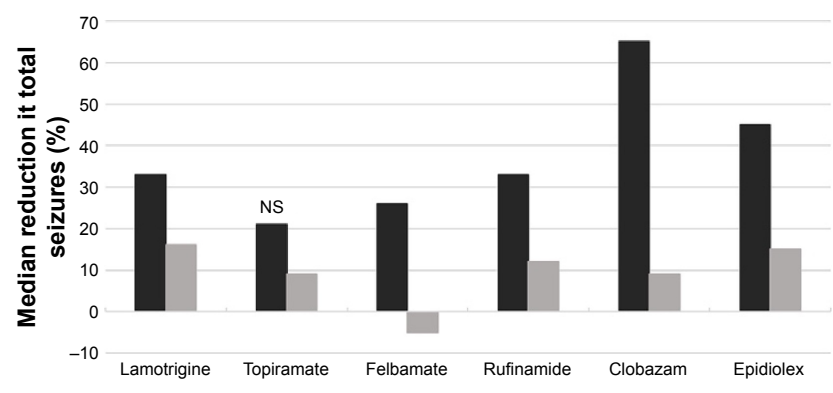

Figure 2 Total seizure efficacy.

Notes: Percentage of reduction in total seizures in patients with Lennox-Gastaut syndrome noted from class I or class II studies of approved antiseizure medications. Dark bars represent study drug results, with corresponding placebo response in light bars. Modified from Pediatr Neurol, 47(3), VanStraten AF, Ng YT. Update on the management of Lennox-Gastaut syndrome. I53-161, Copyright (C) 2012 Elsevier Inc. All rights reserved, with permission from Elsevier."

Abbreviation: NS, not significant.

seizures encompass atonic, tonic or major myoclonic seizures. However, therapies better suited for tonic seizures may worsen myoclonic seizures (ie, lamotrigine). Most studies examine mixed pediatric and adult patients; yet, LGS tends to have a longitudinal progression with variable seizures and treatment resistance over time. In fact, a recent Cochrane review concluded that a meta-analysis was not possible from the currently available data due to the heterogeneities between study populations and outcome measures in the seven class I studies of lamotrigine, topiramate, felbamate and rufinamide for adjunctive therapy for LGS (Figures 2-4 represent a summary of the level I or II evidence). ${ }^{8}$

\section{Medical therapies - available treatments}

Medical management is guided not by comparative drug studies but by physician preference, experience and adjunctive treatment studies with varying levels of evidence. Polytherapy is often required. Based on expert opinion and practice, valproate, topiramate and lamotrigine are considered as first-line

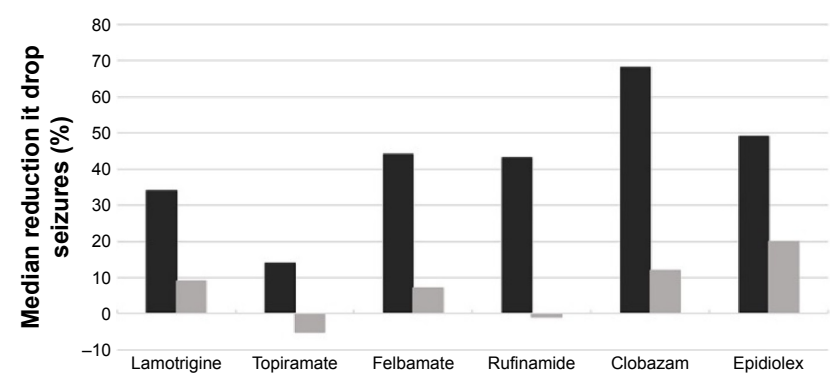

Figure 3 Drop seizure efficacy.

Notes: Percentage of reduction in drop seizures in patients with Lennox-Gastaut syndrome noted from class I or class II studies of approved antiseizure medications. Dark bars represent study drug results, with corresponding placebo response in light bars. Modified from Pediatr Neurol, 47(3), VanStraten AF, Ng YT. Update on the management of Lennox-Gastaut syndrome. I53-16I, Copyright (C) 2012 Elsevier Inc. All rights reserved, with permission from Elsevier."

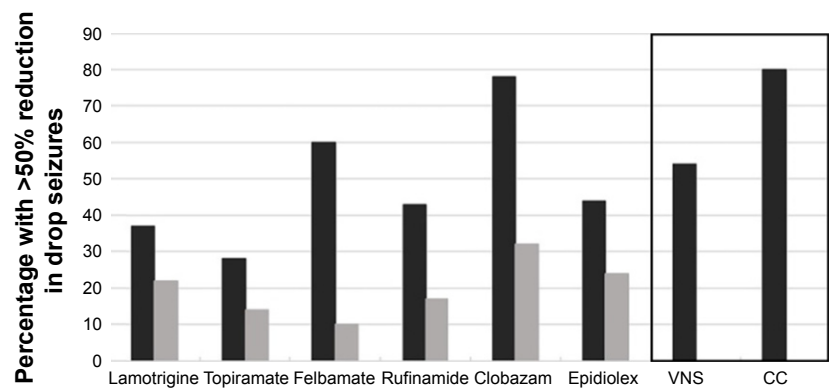

Figure 4 Drop seizure responders.

Notes: Percentage of subjects with a greater than $50 \%$ reduction in drop seizures in patients with Lennox-Gastaut syndrome noted from class I or class II studies of approved antiseizure medications. The results from a meta-analysis of VNS and CC are in the box to the right. Dark bars represent study intervention results, with corresponding placebo response in light bars.

Abbreviations: VNS, vagal nerve stimulator; CC, corpus callosotomy.

therapies. ${ }^{23}$ After FDA approval for use in the United States, clobazam has become a commonly used early adjunctive treatment for LGS. ${ }^{11}$ More than 100 nonrandomized controlled trials (level III) and case-control or cohort series (level IV) addressing the treatment of LGS have been published. To emphasize higher quality evidence for pharmacotherapy, we present efficacy data solely from level I or level II studies. In addition, we briefly address several other medications without mentioning efficacy data in an attempt to help readers navigate the spectrum of commonly used therapies. ${ }^{24,25} \mathrm{We}$ also describe existing evidence for dietary therapy and epilepsy surgical techniques. Although lower level data may provide important insights, we recommend providers focus on higher quality evidence for the initial management of LGS. We have also shared our experience and expert opinions.

First-generation antiseizure medications remain popular early therapies for the treatment of LGS. Despite its age and lack of level I or level II evidence, valproate remains a mainstay in treatment and is often the first-line medication for LGS..$^{24,25}$ Valproate carries risk of hepatotoxicity and thrombocytopenia, which may worsen with polytherapy (see later in this section). Other first-generation antiseizure medications are less commonly used. Carbamazepine may be helpful for focal or tonic seizures in individuals with LGS related to a focal structural lesion. However, it may exacerbate absence seizures and myoclonic seizures. ${ }^{5}$ Ethosuximide may be effective in controlling atypical absence seizures, negative myoclonus and atonic seizures, but would not be expected to treat generalized tonic or tonic-clonic seizures. ${ }^{26-28}$ Clonazepam may be effective for myoclonic or tonic seizures but is associated with more common side effects compared to clobazam and has more tachyphylaxis. ${ }^{29,30}$ We have found, however, that sometimes with switching clonazepam to clobazam, although there may 
be better seizure control and less sedation, there is increased (resumption of) spasticity in patients with cerebral palsy. Phenobarbital may decrease the convulsive seizures but exacerbate absences. ${ }^{5}$

Lamotrigine, topiramate, felbamate and levetiracetam are second-generation antiseizure medications commonly prescribed for patients with LGS and typically with better tolerability. Levetiracetam demonstrated efficacy in small cohorts of patients with LGS and remains a common choice due to an exemplary safety and tolerability profile. $^{31,32}$

Adjunctive lamotrigine has been studied in a class 1 trial of 169 patients with LGS and demonstrated a $>50 \%$ reduction in seizures in $33 \%$ of patients compared to $16 \%$ in the placebo group. ${ }^{33}$ Overall, seizure reduction was significant $(-32 \%$ vs $-9 \%$ placebo). This included a statistically significant decrease in drop attacks (tonic, atonic and major myoclonic seizures). A higher percentage of patients in the lamotrigine group compared to the placebo group were on valproate (67\% to $50 \%$, respectively). Adverse drug events were similar between study drug and placebo groups. Despite this evidence, lamotrigine should be used with caution in patients with a significant number of myoclonic seizures, as it may cause exacerbations of this seizure type..$^{5,34}$

Topiramate was studied in a class I trial in 98 patients with LGS. ${ }^{35}$ Results did not demonstrate a statistically significant decrease in total seizures ( $-21 \%$ vs $-9 \%)$, although greater than $50 \%$ reduction in major seizures occurred in a larger proportion of patients in the treatment arm $(33 \%$ on topiramate vs $8 \%$ placebo). Drop attacks were reduced with topiramate (14\% reduction vs $5 \%$ increase in placebo group). Although adverse events were common, they did not lead to discontinuation of the study drug. The treatment effect dissipated by 30 months. ${ }^{36}$

High-level evidence also exists for felbamate, although it is less commonly used due to concerns regarding side effects. Felbamate likely has multiple mechanisms of action, including as an antagonist at the N-methyl-D-aspartate receptor. Adjunctive treatment with felbamate in a class 1 study of 73 individuals with LGS significantly reduced all seizure types studied ( $-19 \%$ vs $+4 \%$ placebo), although absences were not evaluated and video monitoring did not demonstrate a difference in the frequency of seizures. ${ }^{37,38}$ Total responders with $>50 \%$ reduction in seizures included $50 \%$ of patients treated with felbamate and $15 \%$ in the placebo arm. Adverse drug events were similar in frequency between the two groups, and global functioning improved. This effect was sustained for 12 months in an open-label study following trial completion. ${ }^{39}$

Although several other studies have demonstrated felbamate effective in LGS, ${ }^{38,40}$ clinicians remain judicious with its use. ${ }^{24,25}$ Postmarketing analysis has revealed felbamate may cause aplastic anemia ( 1 in 5,000-10,000) and hepatic failure ( 1 in 26,000 to 34,000 ). ${ }^{11,41}$ In comparison, the risk of aplastic anemia with carbamazepine is between 1 in 50,000-200,000 and the risk of hepatic failure with valproate ranges from 1 in 500 (children less than 2 years of age), 1 in 12,000 (polytherapy) and 1 in 37,000 (monotherapy). ${ }^{42}$

Newer third-generation antiseizure medications have demonstrated efficacy and tolerability in patients with LGS. Rufinamide is a third-generation antiseizure medication that likely prolongs inactivation of sodium channels. In a placebo-controlled study of adjunctive treatment in 138 individuals with LGS, the overall median of total seizures was significantly reduced (33\% vs 12\%). ${ }^{43}$ Drop seizures, identified as tonic-atonic seizures, were reduced ( $-43 \%$ vs $+1 \%$ placebo). Somnolence and vomiting were more common in the treatment group, and $8 \%$ of patients receiving rufinamide withdrew due to side effects. Rufinamide was safe and well tolerated in pediatric subjects. Results from the interim analysis demonstrate that the safety and pharmacokinetic profile of rufinamide in subjects with LGS aged between 1 and 4 years is comparable to that in subjects aged $>4$ years. ${ }^{44}$

Clobazam is a 1,5-benzodiazepine, which binds type A gamma-aminobutyric acid $\left(\mathrm{GABA}_{\mathrm{A}}\right)$ receptors, enhancing the inhibitory effect of GABA on neuron excitability. In a class II study of 238 individuals with LGS treated with 0.25 , 0.5 or $1 \mathrm{mg} / \mathrm{kg} / \mathrm{d}$ of clobazam vs placebo, overall responders with $>50 \%$ seizure reduction were $43 \%, 59 \%$ and $78 \%$, respectively, compared to $32 \%$ for placebo. ${ }^{45}$ The median reduction in seizures in the high-dose group was $65 \%$ vs $9 \%$ in the placebo group. Drop seizures were reduced by $68 \%$ in the $1 \mathrm{mg} / \mathrm{kg} / \mathrm{d}$ clobazam arm compared to $12 \%$ in the placebo group. Common adverse events related to clobazam included somnolence, pyrexia, lethargy, drooling and constipation and severe adverse events occurred in $7 \%$ of patients. This was a class II, as opposed to class 1 study because only $\sim 79 \%$ of the patients with intention to treat analysis completed the study ( $>80 \%$ of patients needed to be a class 1 study); the largest LGS study to date.

Zonisamide likely has multiple mechanisms of action, including reducing inward T-type $\mathrm{Ca}^{2+}$ currents, blocking sodium channels and facilitating dopaminergic and serotonergic neurotransmission. An open-label adjunctive 
study of 62 patients revealed some effect on seizures with adverse events of anorexia and somnolence. ${ }^{46}$ Vigabatrin increases available GABA by irreversibly inhibiting GABA transaminase. In an open-label study of adjunctive treatment combined with valproate in 20 patients with LGS, several children had a reduction in seizures. ${ }^{47}$ Despite encouraging initial data, it is not commonly used due to possible worsening of absences and myoclonic jerks and the risk of irreversible visual field constriction. . $^{54,25}$

Hormonal and immunomodulatory therapies have a limited role in the treatment of LGS due to significant side effects and barriers to use. Corticosteroids and adrenocorticotropic hormone may be helpful during severe seizure exacerbations but cannot be used for prolonged periods due to serious side effects and suppression of the hypothalamic-pituitary-adrenal axis. ${ }^{5,48}$ Intravenous (IV) immunoglobulin was evaluated as adjunctive, open-label treatment in 12 children with LGS with some individuals achieving improvement in seizure control. ${ }^{49}$ Another open-label study of IV methylprednisolone combined with a slow wean of oral prednisolone in 17 children with epileptic encephalopathies, including LGS, resulted in seizure freedom in several patients and overall improvement in seizures in a portion of individuals 6-month posttreatment. ${ }^{50}$ These options may prove useful in select patients with severe exacerbations of seizures.

\section{Dietary therapies}

Dietary therapies, including the classic ketogenic diet and its variants, have demonstrated efficacy in multiple epileptic syndromes. ${ }^{51}$ The mechanisms of action are incompletely understood, but likely decrease neuronal excitability through carbohydrate reduction, potassium channel activation, inhibition of the mammalian target of rapamycin (mTOR) pathway and inhibition of glutamatergic-mediated neuronal excitability. ${ }^{52}$ A randomized, blinded cross-over study of 20 children with LGS over a 6-day period did not produce a significant reduction in EEG-identified or parent-reported (median decrease of 1.5 seizures in the ketogenic arm, $P=0.07$ ) seizures, although some children in the nonketogenic arm had remained in ketosis and likely altered the validity of the comparison. ${ }^{53}$ An extension of this study demonstrated a reduction in seizures that persisted at 12 months. ${ }^{54}$ A study of the ketogenic diet in 71 children with LGS demonstrated a $>50 \%$ reduction in total seizures in $51 \%$ of patients, including a $>90 \%$ reduction in seizures in nearly one-quarter of those treated. ${ }^{55}$ Although effective, the use of these therapies remains limited due to difficulty restricting the dietary habits of individuals with LGS.

\section{Surgical therapy}

Surgical therapy for individuals with treatment resistant epilepsy remains the most successful approach for long-term seizure control. LGS has historically been described as an incurable epileptic encephalopathy due to the often diffuse or generalized EEG findings and intractable seizures despite aggressive medical management. ${ }^{56}$ However, numerous advances in neuroimaging and neurophysiology have enabled better identification and treatment of epileptogenic zones. ${ }^{57}$ Resective surgery may benefit those with focal lesions, such as dysplasias, hamartomas or infarcts. A retrospective cohort of 27 children and adolescents with LGS (85\% lesional on magnetic resonance imaging [MRI]) undergoing resective epilepsy surgery demonstrated seizure freedom in $60 \%$ and improvement in another $15 \%$ with a mean follow-up of 33 months. ${ }^{58}$ In two other case reports, individuals with LGS secondary to focal cortical dysplasias ${ }^{59}$ or heterotopia ${ }^{60}$ were seizure free following resective surgery. In 21 patients with LGS secondary to hypothalamic hamartoma, 95\% had a greater than $50 \%$ reduction in seizures, with $24 \%$ seizure free following surgery. ${ }^{15}$ Initial results can be encouraging; however, the effect of surgical resection tends to wane similar to other therapies. Focal resective surgery initially resulted in Engel class 1 outcomes in 10/15 patients, although seizure freedom in adulthood only occurred in $3 / 15 .{ }^{13}$

While carefully selected patients with LGS will be candidates for resective surgery aimed at curing their epilepsy, many others may benefit from a surgical approach. Palliative surgical approaches are commonly used to optimize seizure control in individuals with LGS, especially those with the significant morbidity of drop attacks. The vagus nerve stimulator (VNS) implantation is a minimally invasive, reversible procedure without the risk of craniotomy with well-established efficacy in treating epilepsy despite an unknown mechanism of action. ${ }^{61}$ Corpus callosotomy (CC) involves severing the main interhemispheric white matter tract through a craniotomy, making this a more invasive surgical option.

A meta-analysis of CC compared to VNS included analysis of 203 VNS patients and $135 \mathrm{CC}$ patients. ${ }^{62}$ The authors found that a larger number of patients with $\mathrm{CC}$ had a $>50 \%$ reduction in atonic seizures $(80 \%$ vs $54 \%$ with VNS, $P<0.05)$. All other seizure types were statistically similar. Importantly, however, only $19 \%$ of included patients had complete $\mathrm{CC}$, which has demonstrated better efficacy in controlling generalized tonic-clonic, astatic, absence, myoclonic and focal onset seizures with dyscognitive features when compared with anterior $2 / 3$ callosotomy. ${ }^{63}$ Between 

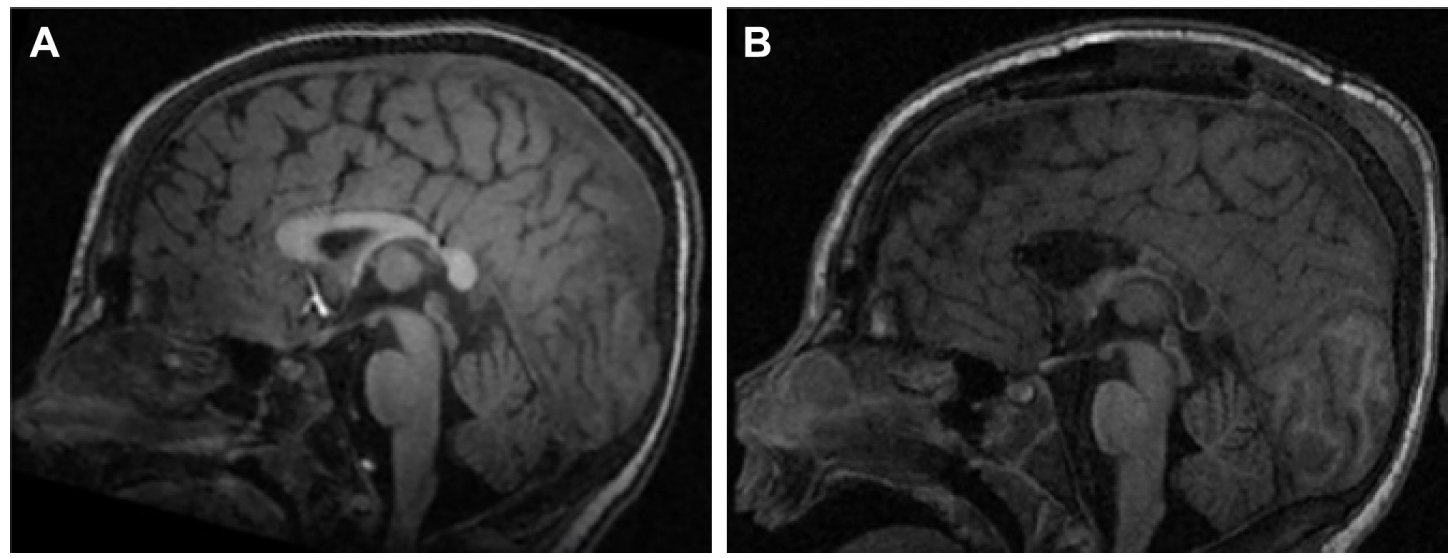

Figure $\mathbf{5}$ Complete corpus callosotomy.

Note: Magnetic resonance imaging $(\mathbf{A})$ before and $(\mathbf{B})$ after complete corpus callosotomy in an individual with Lennox-Gastaut syndrome.

$61 \%$ and $85 \%$ of patients achieve an $80 \%$ or greater reduction in drop seizures, including $48 \%$ of individuals free of drop seizures following the procedure. ${ }^{64,65}$ These data indicate that complete $\mathrm{CC}$ may provide superior seizure control over anterior 2/3 CC (Figure 5).

The safety and tolerability of VNS is favorable, with side effects including hoarseness, coughing, tingling in the throat and voice changes occurring in less than $4 \%$ of individuals. ${ }^{61,66}$ Complications from CC are more common, occurring in $\sim 8 \%$ of patients and include perioperative complications such as infarct or meningitis or transient neurological deficits such as akinesia, mutism, hemiparesis, disconnection syndrome and constructional apraxia. ${ }^{62,63}$ As with all surgical procedures, complications may vary between centers.

\section{Synergy}

Several treatment options have been studied in combination and may provide synergies. A retrospective analysis of 30 children treated with both VNS and dietary therapy demonstrated a $>50 \%$ reduction in seizures in $70 \%$ of patients. ${ }^{67}$ Distinct surgical approaches have been combined in individuals with LGS. In a study including 23 children receiving simultaneous resective surgery and $\mathrm{CC}$ demonstrated a greater improvement in quality of life and intelligence quotient. Seizure outcomes were not statistically different compared to medical or resection-only procedures ${ }^{68}$ In other instances, CC may lead to lateralization or localization of diffuse EEG ictal and/or interictal findings. ${ }^{69}$ The combination of the palliative procedures VNS and CC in 10 individuals with LGS provided a greater than $50 \%$ reduction in seizures in $60 \%$ of patients and $2 / 10$ demonstrating seizure freedom at 12 months. ${ }^{70}$ Drop attacks were reduced significantly in $77 \%$. If pharmacotherapy fails, these two surgical approaches in combination or coupled with dietary therapy may be reasonable options.

Creating a treatment algorithm capable of guiding a provider through treatment options for patients with LGS, a complex and heterogeneous severe epilepsy, remains a challenge. However, we present an example workflow that the practitioner may use as a framework (Figure 6). We recommend strongly considering clobazam, topiramate or rufinamide initially based on efficacy and side effect profiles. Lamotrigine or felbamate may be effective, but caution should be exercised regarding potential exacerbation of myoclonic seizures and small risk of Stevens-Johnson syndrome in the former and potential life-threatening adverse reactions in the latter. Levetiracetam and valproate remain common choices, but strong supportive data are lacking. Finally, it is reasonable to pursue potential surgery options and dietary or medication therapies after failure of two or more antiseizure medications.

\section{Investigational and other therapies}

Currently approved antiseizure therapies have failed to provide long-lasting seizure control for most individuals with LGS. Therapies under investigation include cannabinoids, fenfluramine and transcranial direct current stimulation.

Cannabidiol (CBD) is one of the two major neuroactive compounds in cannabis and has been the subject of intense interest as a new therapeutic option for patients with LGS. The antiseizure mechanisms of CBD are not definitively known, although studies have implicated G-coupled protein receptor protein 55 (GPR55), transient receptor potential of melastatin type 8 or ankyrin type 1 channels, the $5-\mathrm{HT}_{1 \mathrm{a}}$ receptor or the alpha3 and alpha1 glycine receptors as playing a role in reducing neuronal excitability. ${ }^{71}$ 


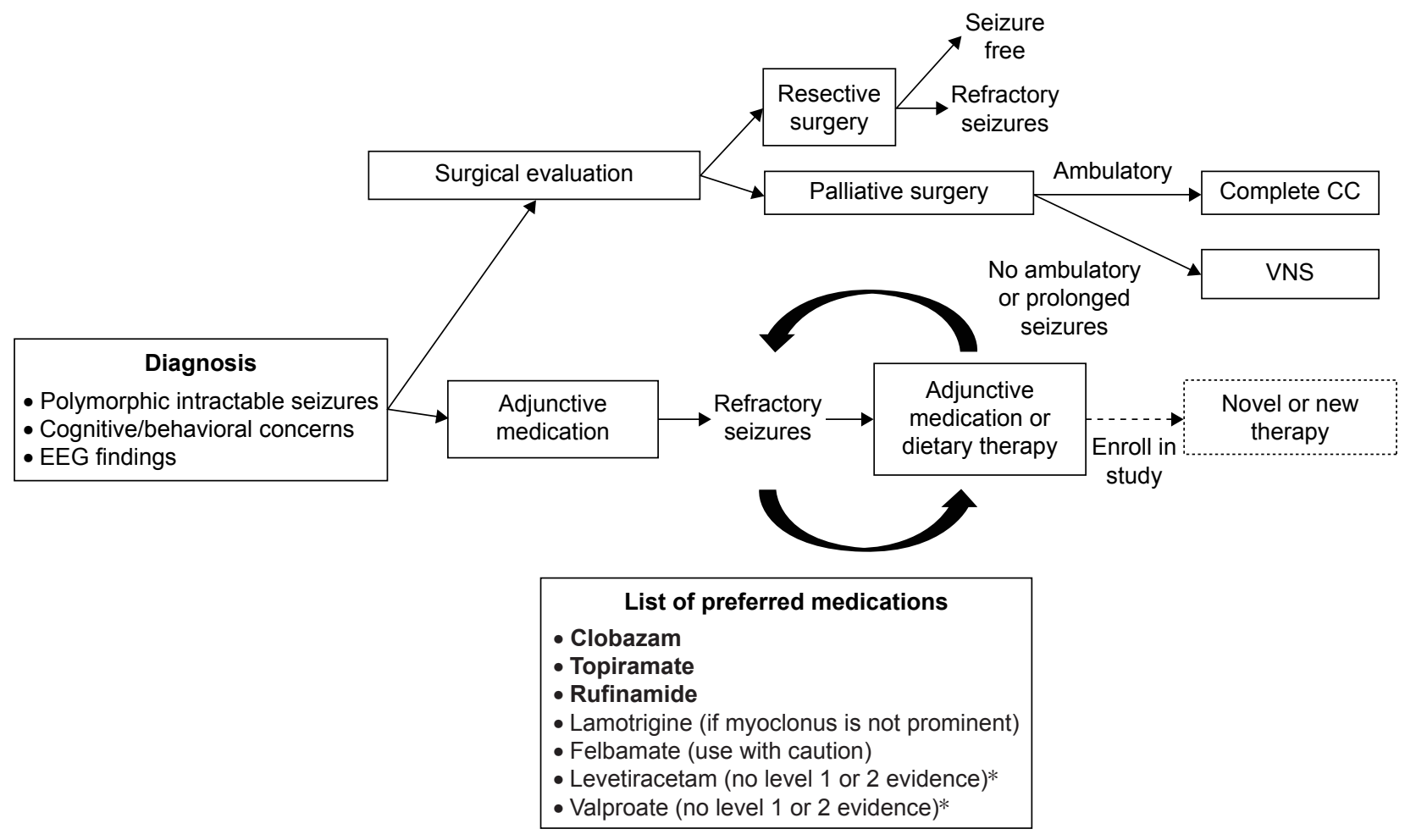

Figure 6 Proposed management of Lennox-Gastaut syndrome.

Notes: First-tier preferred medications are in bold. *Commonly used medications without level I or 2 evidence.

Abbreviations: CC, corpus callosotomy; EEG, electroencephalogram; VNS, vagal nerve stimulator.

Several parental surveys demonstrated a perceived decrease in seizures of $>50 \%$ in over $80 \%$ of individuals with LGS after administering CBD-enriched compounds with good tolerability. ${ }^{72-74}$ Based on these basic science and anecdotal data, recent open-label and phase 3 studies have been conducted utilizing Epidiolex, a purified CBD extract from the marijuana plant. Efficacy data from the open-label add-on study evaluated 30 individuals with LGS of 137 total patients with epilepsy. ${ }^{75}$ Post hoc analysis of this subgroup revealed a median reduction of $37 \%$ in motor seizures and $37 \%$ of patients had a $>50 \%$ reduction in total seizures. Drop seizures were reduced by a median of $69 \%$ in the 14 patients with atonic seizures. Common adverse events in $>10 \%$ of subjects included somnolence, diarrhea, fatigue and anorexia and serious adverse events possibly related to CBD use occurred in $12 \%$. Of 162 (3\%) patients, 5 discontinued treatment due to adverse events.

Data from a recent randomized, double-blind, placebocontrolled trial of CBD as add-on therapy in 171 individuals with LGS demonstrated further promising results. ${ }^{76}$ During the treatment period after a 4-week titration, median reduction in total seizures was $45 \%$ vs $15 \%$ in the placebo group. Drop seizures were reduced by a median of $49 \%$ compared to $20 \%$ in the placebo group. Adverse events occurring in greater than $10 \%$ of patients included diarrhea, somnolence, pyrexia, decreased appetite and vomiting. Serious adverse events occurred in $23 \%$ of patients on CBD (vs $5 \%$ on placebo) and led to withdrawal in $14 \%$ in the CBD group (vs $1 \%$ on placebo).

Pharmacokinetic data and post hoc analysis of data from the open-label study implicate an interaction between CBD and clobazam, with possible synergy. CBD inhibits CYP2C19, which increases the concentrations of the antiseizure metabolite, $\mathrm{N}$-desmethyl clobazam. ${ }^{77} \mathrm{~A}$ greater number of patients taking this combination demonstrated a $>50 \%$ reduction in motor seizures (51\% on combination therapy vs $27 \%$ in patients not taking clobazam). ${ }^{75}$ Although there may be synergy between these two drugs, concerns arose if one of the main reasons for the overall positive results is due to increasing N-desmethyl clobazam levels. Valproate and CBD may also synergize both in treatment and adverse events. More patients taking the combination of valproate and $\mathrm{CBD}$ also had a $>50 \%$ reduction in seizures compared to other medication combinations ( $54 \%$ vs $33 \%$, respectively), although this finding was not significant after multiple logistic regression analysis. ${ }^{75}$ Recent data revealed increases in aspartate aminotransferase and alanine aminotransferase in $28 \%$ of patients treated with CBD, $80 \%$ of whom were taking 
concurrent valproate. Further studies are needed to better characterize the interactions between traditional antiseizure medications and CBD.

Fenfluramine is a drug previously marketed under the name Fen-phen as a weight loss medication no longer used due to postmarketing surveillance identifying significant heart disease and pulmonary hypertension. Drug effects include release of serotonin and have demonstrated antiseizure effects in rat entorhinal cortex. ${ }^{78}$ An open-label prospective adjunctive trial in individuals with Dravet syndrome, an epileptic encephalopathy similar in severity to LGS revealed a median reduction of seizures of $75 \%$, with seven of nine patients experiencing a $>50 \%$ reduction in major motor seizures. No major side effects were noted after a median duration of 1.5 years. ${ }^{79}$ Currently, an adjunctive open-label study for individuals with LGS is underway recruiting patients. ${ }^{80}$

Transcranial direct current stimulation is a nonpharmacologic approach to seizure management with encouraging, but limited, data in humans. The delivery of negative stimulation leads to hyperpolarization of the neuronal membranes and may provide antiseizure effects. ${ }^{81}$ Two children with LGS and focal seizures emanating from motor cortex were treated with adjunctive transcranial direct current stimulation or sham with a subsequent decrease in seizures with a $90 \%$ reduction at 1 week and $72 \%$ reduction at 3 weeks following treatment. ${ }^{82}$

When treating patients with refractory epilepsy or even mild epilepsy, both the caregivers and caretakers can be blinded by the goal of complete cessation or control of seizures. This goal is often unattainable, and its pursuit may result in undesirable side effects which may diminish the quality of life for both the patient and family. Hence, a treatment like the VNS although very unlikely to make a refractory LGS patient seizure free, may well have many positive cognitive and mood effects and also result in lowering of medications. ${ }^{83}$ By definition, patients with LGS have intellectual disability and often they have cerebral palsy as well, and appropriate schooling and management of associated comorbidities (eg, spasticity, abnormal sleep patterns) are often neglected. Hence, an excellent primary pediatrician who can help coordinate comprehensive care and social aspects would be very helpful. The stigma and different attitudes toward these children or adults cannot be understated. ${ }^{11}$ Often the schooling system, with teachers and nurses not allowing LGS children with seizures to attend, or possibly even worse, calling a parent or a caregiver to take them home or an ambulance every time there is a routine seizure is more restricting than the seizures or disease state itself. In fact, we often encourage all patients with epilepsy to be allowed to participate in all regular activities as tolerated with the only real restriction of lifeguard supervision whenever in water or swimming. We, the expert clinicians, need to work and communicate better and more with these groups of people who care for the patients for many hours each day.

\section{Conclusion}

LGS remains an incredible therapeutic challenge for practitioners and poses immense risk and morbidity for afflicted individuals well into adulthood. Diverse etiologies, transient response to current therapies and various seizure types persistently create obstacles to lasting relief from epilepsy. Comparative data regarding therapeutic options are nonexistent, but several medical treatments are substantiated by high-level evidence and may provide relief in combination. Dietary therapy and epilepsy surgery may palliate the disease and rarely provide seizure freedom. Novel pharmacologic and noninvasive neurophysiologic therapeutic approaches are currently in both early- and late-stage development and may provide further relief from symptoms. CBD works for the treatment of seizures, although apparently not dramatically better than any of the currently other available treatments; it will likely obtain FDA approval soon. This medication will be widely used and sought after, and it is our belief that many patients and families who are antidrugs view this as an alternative, natural therapy. Furthermore, there are major financial and business implications with the earliest studies on plant extract CBD, while at least one other company is generating synthetic CBD. Ultimately, direct comparative data are desperately needed to guide best practice for these patients.

\section{Disclosure}

Yu-Tze Ng, MBBS, is on the Advisory Boards of Mallinckrodt Pharmaceuticals, Inc., Eisai Inc., Lundbeck Inc. and Zogenix Inc. and the Speakers' Bureaus of Sunovion Pharmaceuticals, Inc., Lundbeck, Inc., Cyberonics Inc., UCB Pharma and Eisai Inc. The authors report no other conflicts of interest in this work.

\section{References}

1. Gastaut H, Roger J, Soulayrol R, et al. Childhood epileptic encephalopathy with diffuse slow spike-waves (otherwise known as "petit mal variant") or Lennox syndrome. Epilepsia. 1966;7(2):139-179.

2. Berg AT, Berkovic SF, Brodie MJ, et al. Revised terminology and concepts for organization of seizures and epilepsies: report of the ILAE Commission on Classification and Terminology, 2005-2009. Epilepsia. 2010;51(4):676-685.

3. Engel J; International League Against Epilepsy (ILAE). A proposed diagnostic scheme for people with epileptic seizures and with epilepsy: report of the ILAE Task Force on Classification and Terminology. Epilepsia. 2001;42(6):796-803. 
4. Rantala H, Putkonen T. Occurrence, outcome, and prognostic factors of infantile spasms and Lennox-Gastaut syndrome. Epilepsia. 1999; 40(3):286-289.

5. Panayiotopoulos CP. A Clinical Guide to Epileptic Syndromes and Their Treatment: Based on the ILAE Classifications and Practice Parameter Guidelines. Rev. 2nd ed. London: Springer; 2010.

6. Gastaut H, Gastaut JL, Gonçalves e Silva GE, Fernandez Sanchez GR. Relative frequency of different types of epilepsy: a study employing the classification of the International League against epilepsy. Epilepsia. 1975;16(3):457-461.

7. Trevathan E, Murphy CC, Yeargin-Allsopp M. Prevalence and descriptive epidemiology of Lennox-Gastaut syndrome among Atlanta children. Epilepsia. 1997;38(12):1283-1288.

8. Hancock EC, Cross JH. Treatment of Lennox-Gastaut syndrome. Cochrane Database Syst Rev. 2013;(2):CD003277.

9. Bourgeois BFD, Douglass LM, Sankar R. Lennox-Gastaut syndrome: a consensus approach to differential diagnosis. Epilepsia. 2014; 55(suppl 4):4-9.

10. Gibson PA. Lennox-Gastaut syndrome: impact on the caregivers and families of patients. J Multidiscip Healthc. 2014;7:441-448.

11. VanStraten AF, Ng YT. Update on the management of Lennox-Gastaut syndrome. Pediatr Neurol. 2012;47(3):153-161.

12. Tomson T, Surges R, Delamont R, Haywood S, Hesdorffer DC. Who to target in sudden unexpected death in epilepsy prevention and how? Risk factors, biomarkers, and intervention study designs. Epilepsia. 2016;57(suppl 1):4-16.

13. Kim HJ, Kim HD, Lee JS, Heo K, Kim D-S, Kang H-C. Long-term prognosis of patients with Lennox-Gastaut syndrome in recent decades. Epilepsy Res. 2015;110:10-19.

14. Blume WT, David RB, Gomez MR. Generalized sharp and slow wave complexes. Associated clinical features and long-term follow-up. Brain J Neurol. 1973;96(2):289-306.

15. Pati S, Deep A, Troester MM, Kossoff EH, Ng Y-T. Lennox-Gastaut syndrome symptomatic to hypothalamic hamartoma: evolution and longterm outcome following surgery. Pediatr Neurol. 2013;49(1):25-30.

16. Kim J, Kundu M, Viollet B, Guan K-L. AMPK and mTOR regulate autophagy through direct phosphorylation of Ulk1. Nat Cell Biol. 2011; 13(2):132-141.

17. Epi4K Consortium; Epilepsy Phenome/Genome Project; Allen AS, et al. De novo mutations in epileptic encephalopathies. Nature. 2013; 501(7466):217-221.

18. Nieh SE, Sherr EH. Epileptic encephalopathies: new genes and new pathways. Neurother J Am Soc Exp Neurother. 2014;11(4): 796-806.

19. Oguni H, Hayashi K, Osawa M. Long-term prognosis of Lennox-Gastaut syndrome. Epilepsia. 1996;37(suppl 3):44-47.

20. Autry AR, Trevathan E, Van Naarden Braun K, Yeargin-Allsopp M. Increased risk of death among children with Lennox-Gastaut syndrome and infantile spasms. J Child Neurol. 2010;25(4):441-447.

21. Blume WT. Pathogenesis of Lennox-Gastaut syndrome: considerations and hypotheses. Epileptic Disord. 2001;3(4):183-196.

22. Goldsmith IL, Zupanc ML, Buchhalter JR. Long-term seizure outcome in 74 patients with Lennox-Gastaut syndrome: effects of incorporating MRI head imaging in defining the cryptogenic subgroup. Epilepsia. 2000;41(4):395-399.

23. Lemmon ME, Kossoff EH. New treatment options for Lennox-Gastaut syndrome. Curr Treat Options Neurol. 2013;15(4):519-528.

24. Wheless JW, Clarke DF, Carpenter D. Treatment of pediatric epilepsy: expert opinion, 2005. J Child Neurol. 2005;20(suppl 1):S1-S56. quiz S59-S60.

25. Wheless JW, Clarke DF, Arzimanoglou A, Carpenter D. Treatment of pediatric epilepsy: European expert opinion, 2007. Epileptic Disord. 2007;9(4):353-412.

26. Arzimanoglou A, French J, Blume WT, et al. Lennox-Gastaut syndrome: a consensus approach on diagnosis, assessment, management, and trial methodology. Lancet Neurol. 2009;8(1):82-93.

27. Snead OC, Hosey LC. Treatment of epileptic falling spells with ethosuximide. Brain Dev. 1987;9(6):602-604.
28. Oguni H, Uehara T, Tanaka T, Sunahara M, Hara M, Osawa M. Dramatic effect of ethosuximide on epileptic negative myoclonus: implications for the neurophysiological mechanism. Neuropediatrics. 1998;29(1):29-34.

29. Cortez MA, McKerlie C, Snead OC. A model of atypical absence seizures: EEG, pharmacology, and developmental characterization. Neurology. 2001;56(3):341-349.

30. Sankar R, Chung S, Perry MS, Kuzniecky R, Sinha S. Clinical considerations in transitioning patients with epilepsy from clonazepam to clobazam: a case series. J Med Case Reports. 2014;8:429.

31. Chhun S, Troude P, Villeneuve N, et al. A prospective open-labeled trial with levetiracetam in pediatric epilepsy syndromes: continuous spikes and waves during sleep is definitely a target. Seizure. 2011;20(4):320-325.

32. De Los Reyes EC, Sharp GB, Williams JP, Hale SE. Levetiracetam in the treatment of Lennox-Gastaut syndrome. Pediatr Neurol. 2004; 30(4):254-256.

33. Motte J, Trevathan E, Arvidsson JF, Barrera MN, Mullens EL, Manasco P. Lamotrigine for generalized seizures associated with the Lennox-Gastaut syndrome. Lamictal Lennox-Gastaut Study Group. N Engl J Med. 1997;337(25):1807-1812.

34. Guerrini R, Dravet C, Genton P, Belmonte A, Kaminska A, Dulac O. Lamotrigine and seizure aggravation in severe myoclonic epilepsy. Epilepsia. 1998;39(5):508-512.

35. Sachdeo RC, Glauser TA, Ritter F, Reife R, Lim P, Pledger G. A doubleblind, randomized trial of topiramate in Lennox-Gastaut syndrome. Topiramate YL Study Group. Neurology. 1999;52(9):1882-1887.

36. Grosso S, Franzoni E, Iannetti P, et al. Efficacy and safety of topiramate in refractory epilepsy of childhood: long-term follow-up study. J Child Neurol. 2005;20(11):893-897.

37. Efficacy of felbamate in childhood epileptic encephalopathy (LennoxGastaut syndrome). The Felbamate Study Group in Lennox-Gastaut Syndrome. N Engl J Med. 1993;328(1):29-33.

38. Dodson WE. Felbamate in the treatment of Lennox-Gastaut syndrome: results of a 12-month open-label study following a randomized clinical trial. Epilepsia. 1993;34(suppl 7):S18-S24.

39. Jensen PK. Felbamate in the treatment of Lennox-Gastaut syndrome. Epilepsia. 1994;35(suppl 5):S54-S57.

40. Siegel H, Kelley K, Stertz B, et al. The efficacy of felbamate as add-on therapy to valproic acid in the Lennox-Gastaut syndrome. Epilepsy Res. 1999;34(2-3):91-97.

41. Pellock JM, Faught E, Leppik IE, Shinnar S, Zupanc ML. Felbamate: consensus of current clinical experience. Epilepsy Res. 2006;71(2-3): 89-101.

42. Zaccara G. The treatment of epilepsy. In: Shovon SD, Perucca E, Engel J Jr, editors. The Treatment of Epilepsy. 3rd ed. Oxford: John Wiley \& Sons; 2009:459-474, 685-698.

43. Glauser T, Kluger G, Sachdeo R, Krauss G, Perdomo C, Arroyo S. Rufinamide for generalized seizures associated with Lennox-Gastaut syndrome. Neurology. 2008;70(21):1950-1958.

44. Arzimanoglou A, Ferreira JA, Satlin A, et al. Safety and pharmacokinetic profile of rufinamide in pediatric patients aged less than 4 years with Lennox-Gastaut syndrome: an interim analysis from a multicenter, randomized, active-controlled, open-label study. Eur J Paediatr Neurol. 2016;20(3):393-402.

45. Ng YT, Conry JA, Drummond R, Stolle J, Weinberg MA; OV-1012 Study Investigators. Randomized, phase III study results of clobazam in Lennox-Gastaut syndrome. Neurology. 2011;77(15):1473-1481.

46. You SJ, Kang H-C, Kim HD, Lee HS, Ko T-S. Clinical efficacy of zonisamide in Lennox-Gastaut syndrome: Korean multicentric experience. Brain Dev. 2008;30(4):287-290.

47. Feucht M, Brantner-Inthaler S. Gamma-vinyl-GABA (vigabatrin) in the therapy of Lennox-Gastaut syndrome: an open study. Epilepsia. 1994; 35(5):993-998.

48. Ferrie CD, Patel A. Treatment of Lennox-Gastaut Syndrome (LGS). Eur J Paediatr Neurol. 2009;13(6):493-504.

49. van Engelen BG, Renier WO, Weemaes CM, Strengers PF, Bernsen PJ, Notermans SL. High-dose intravenous immunoglobulin treatment in cryptogenic West and Lennox-Gastaut syndrome; an add-on study. Eur J Pediatr. 1994;153(10):762-769. 
50. Almaabdi KH, Alshehri RO, Althubiti AA, et al. Intravenous methylprednisolone for intractable childhood epilepsy. Pediatr Neurol. 2014;50(4):334-336.

51. Levy RG, Cooper PN, Giri P. Ketogenic diet and other dietary treatments for epilepsy. Cochrane Database Syst Rev. 2012;(3):CD001903.

52. Danial NN, Hartman AL, Stafstrom CE, Thio LL. How does the ketogenic diet work? Four potential mechanisms. J Child Neurol. 2013; 28(8):1027-1033.

53. Freeman JM, Vining EPG, Kossoff EH, Pyzik PL, Ye X, Goodman SN. A blinded, crossover study of the efficacy of the ketogenic diet. Epilepsia. 2009;50(2):322-325.

54. Freeman JM. The ketogenic diet: additional information from a crossover study. J Child Neurol. 2009;24(4):509-512.

55. Lemmon ME, Terao NN, Ng Y-T, Reisig W, Rubenstein JE, Kossoff EH. Efficacy of the ketogenic diet in Lennox-Gastaut syndrome: a retrospective review of one institution's experience and summary of the literature. Dev Med Child Neurol. 2012;54(5):464-468.

56. Pellock JM, Wheless JW. Introduction: recommendations regarding management of patients with Lennox-Gastaut syndrome. Epilepsia. 2014;55(suppl 4):1-3.

57. Douglass LM, Salpekar J. Surgical options for patients with LennoxGastaut syndrome. Epilepsia. 2014;55(suppl 4):21-28.

58. Lee YJ, Kang H-C, Lee JS, et al. Resective pediatric epilepsy surgery in Lennox-Gastaut syndrome. Pediatrics. 2010;125(1):e58-e66.

59. You SJ, Lee J-K, Ko T-S. Epilepsy surgery in a patient with LennoxGastaut syndrome and cortical dysplasia. Brain Dev. 2007;29(3): 167-170.

60. Ng Y-T, Pati S, Fesler JR. Lennox-Gastaut syndrome may be a curable, reversible epileptic encephalopathy. Epilepsia. 2015;56(3):499-500.

61. Elliott RE, Morsi A, Kalhorn SP, et al. Vagus nerve stimulation in 436 consecutive patients with treatment-resistant epilepsy: long-term outcomes and predictors of response. Epilepsy Behav. 2011;20(1): 57-63.

62. Lancman G, Virk M, Shao H, et al. Vagus nerve stimulation vs corpus callosotomy in the treatment of Lennox-Gastaut syndrome: a metaanalysis. Seizure. 2013;22(1):3-8.

63. Kasasbeh AS, Smyth MD, Steger-May K, Jalilian L, Bertrand M, Limbrick DD. Outcomes after anterior or complete corpus callosotomy in children. Neurosurgery. 2014;74(1):17-28.

64. Maehara T, Shimizu H. Surgical outcome of corpus callosotomy in patients with drop attacks. Epilepsia. 2001;42(1):67-71.

65. Nei M, O’Connor M, Liporace J, Sperling MR. Refractory generalized seizures: response to corpus callosotomy and vagal nerve stimulation. Epilepsia. 2006;47(1):115-122.

66. Ramsay RE, Uthman BM, Augustinsson LE, et al. Vagus nerve stimulation for treatment of partial seizures: 2 . Safety, side effects, and tolerability. First International Vagus Nerve Stimulation Study Group. Epilepsia. 1994;35(3):627-636.

67. Kossoff EH, Pyzik PL, Rubenstein JE, et al. Combined ketogenic diet and vagus nerve stimulation: rational polytherapy? Epilepsia. 2007; 48(1):77-81.

68. Ding P, Liang S, Zhang S, Zhang J, Hu X, Yu X. Resective surgery combined with corpus callosotomy for children with non-focal lesional Lennox-Gastaut syndrome. Acta Neurochir (Wien). 2016;158(11): 2177-2184.
69. Hur YJ, Kang H-C, Kim DS, Choi SR, Kim HD, Lee JS. Uncovered primary seizure foci in Lennox-Gastaut syndrome after corpus callosotomy. Brain Dev. 2011;33(8):672-677.

70. Katagiri M, Iida K, Kagawa K, et al. Combined surgical intervention with vagus nerve stimulation following corpus callosotomy in patients with Lennox-Gastaut syndrome. Acta Neurochir (Wien). 2016;158(5): 1005-1012.

71. Devinsky O, Cilio MR, Cross H, et al. Cannabidiol: pharmacology and potential therapeutic role in epilepsy and other neuropsychiatric disorders. Epilepsia. 2014;55(6):791-802.

72. Hussain SA, Zhou R, Jacobson C, et al. Perceived efficacy of cannabidiol-enriched cannabis extracts for treatment of pediatric epilepsy: a potential role for infantile spasms and Lennox-Gastaut syndrome. Epilepsy Behav. 2015;47:138-141.

73. Porter BE, Jacobson C. Report of a parent survey of cannabidiolenriched cannabis use in pediatric treatment-resistant epilepsy. Epilepsy Behav. 2013;29(3):574-577.

74. Press CA, Knupp KG, Chapman KE. Parental reporting of response to oral cannabis extracts for treatment of refractory epilepsy. Epilepsy Behav. 2015;45:49-52.

75. Devinsky O, Marsh E, Friedman D, et al. Cannabidiol in patients with treatment-resistant epilepsy: an open-label interventional trial. Lancet Neurol. 2016;15(3):270-278.

76. Thiele EA, Mazurkiewicz-Beldzinska M, Benbadis S, et al. Cannabidiol significantly reduces drop seizure frequency in Lennox-Gastut syndrome: results of a multi-center, randomized, double-blind, placebo controlled trial (GWPCARE4). Abstract from the 2016 Annual American Epilepsy Society; Houston, TX; 2016.

77. Geffrey AL, Pollack SF, Bruno PL, Thiele EA. Drug-drug interaction between clobazam and cannabidiol in children with refractory epilepsy. Epilepsia. 2015;56(8):1246-1251.

78. Gentsch K, Heinemann U, Schmitz B, Behr J. Fenfluramine blocks low$\mathrm{Mg} 2+$-induced epileptiform activity in rat entorhinal cortex. Epilepsia. 2000;41(8):925-928.

79. Schoonjans A, Paelinck BP, Marchau F, et al. Low-dose fenfluramine significantly reduces seizure frequency in Dravet syndrome: a prospective study of a new cohort of patients. Eur J Neurol. 2017;24(2): 309-314.

80. ClinicalTrials.gov. FFA-LGS; 2016. Available from: https://clinicaltrials. gov/ct2/show/NCT02655198?term=lennox\&rank=1. ClinicalTrials.gov identifier NCT02655198. Accessed December 10, 2016.

81. San-Juan D, Morales-Quezada L, Orozco Garduño AJ, et al. Transcranial direct current stimulation in epilepsy. Brain Stimulat. 2015;8(3): 455-464.

82. Auvichayapat N, Sinsupan K, Tunkamnerdthai O, Auvichayapat P. Transcranial direct current stimulation for treatment of childhood pharmacoresistant Lennox-Gastaut Syndrome: A Pilot Study. Front Neurol. 2016;7:66.

83. Grill MF, Ng Y. Dramatic first words spoken in 2 children after vagus nerve stimulation. Semin Pediatr Neurol. 2010;17(1):54-57.
Neuropsychiatric Disease and Treatment

\section{Publish your work in this journal}

Neuropsychiatric Disease and Treatment is an international, peerreviewed journal of clinical therapeutics and pharmacology focusing on concise rapid reporting of clinical or pre-clinical studies on a range of neuropsychiatric and neurological disorders. This journal is indexed on PubMed Central, the 'PsycINFO' database and CAS,
Dovepress

and is the official journal of The International Neuropsychiatric Association (INA). The manuscript management system is completely online and includes a very quick and fair peer-review system, which is all easy to use. Visit http://www.dovepress.com/testimonials.php to read real quotes from published authors. 